\title{
Comportamento clínico e perfil hematológico de cães intoxicados experimentalmente com carbamato (Aldicarb) e submetidos à hemodiálise e hemoperfusão
}

\section{Clinical evaluation and blood profile of dogs experimentally intoxicated by carbamate (Aldicarb) and submitted to hemodialysis and hemoperfusion}

\author{
Priscylla Tatiana Chalfun Guimarães, ${ }^{*}$ Júlio César Cambraia Veado, ${ }^{*}$ Marília Martins Melo, ${ }^{\star}$ Juliana de Oliveira, ${ }^{* \star}$ \\ Marcos Barrouin Melo***
}

\begin{abstract}
Resumo
Técnicas dialíticas são estudadas a fim de se verificar suas reais contribuições no tratamento das mais diversas formas de intoxicações. Esta pesquisa foi realizada com o objetivo de avaliar o comportamento clínico e o perfil hematológico de cães intoxicados com cabamato (Aldicarb) e submetidos a duas diferentes técnicas dialíticas: hemodiálise e hemoperfusão. Quinze cães adultos, sem raça definida foram intoxicados experimentalmente com 4,97mg/kg de peso vivo de Aldicarb, por via oral. Todos os animais, para controle dos efeitos clínicos provocados pela droga, foram medicados 30 minutos e uma hora após a ingestão, com sulfato de atropina e benzodiazepínico (Diazepam), ambos na dose de $1 \mathrm{mg} / \mathrm{kg}$. Esses cães foram divididos em três grupos experimentais, compostos de cinco animais cada, denominados grupos I, II e III. Os animais do grupo II e do grupo III, três horas após administração do Aldicarb foram submetidos a sessões de duas horas de hemodiálise e de hemoperfusão, respectivamente. Os animais do grupo I serviram de controle, não tendo sido submetidos a nenhum tratamento dialítico. A intoxicação provocada pelo aldicarb foi capaz de causar alterações clínicas manifestadas por vômito, sialorréia, diarréia, incontinência urinária, fasciculações e alterações no perfil hematológico relacionadas principalmente a hemoconcentração. Houve leucocitose e aumento no número absoluto de neutrófilos em todos os animais 30 minutos após administração do carbamato. Não foram observadas diferenças clínicas entre os animais do grupo controle e os animais tratados por hemodiálise ou hemoperfusão. Após a sessão de hemoperfusão ocorreu discreta redução no número dos leucócitos, com diminuição dos valores absolutos de eosinófilos, basófilos, monócitos e linfócitos. Nenhuma das técnicas foi efetiva em retirar o Aldicab do organismo.
\end{abstract}

Palavras-chave: cão, intoxicação, carbamato, técnicas dialíticas, hematologia.

\begin{abstract}
The aim of this work was to study the contribution of dialysis techniques, hemodialysis and hemoperfusion, after poisoning by Aldicarb (carbamate), an anticholinesterasic compound, in dogs. Fifteen adult mongrel dogs were experimentally intoxicated by $4.97 \mathrm{mg} / \mathrm{kg}$ of Aldicarb orally. All animals, to prevent and treatment of clinical signs received a standard treatment with atropine sulfate $(1 \mathrm{mg} / \mathrm{kg})$ and benzodiazepine $(1 \mathrm{mg} / \mathrm{kg})$, administered endovenously 30 minutes and one hour after poisoning. The dogs were separated in three groups: group I (control), group II (hemodialysis) and group III (hemoperfusion) with five animals in each. Hemodialysis and hemoperfusion were performed three hours after poisoning, through a double lumen catheter implanted in the jugular vein. Symptoms like vomiting, diarrhea, urinary incontinence and muscle fasciculation were observed after poisoning in all groups. It was observed increase of leucocytes and neutrophils, 30 minutes after carbamate administration. Any clinical difference was observed after therapy with hemodialysis and hemoperfusion. After hemoperfusion occurred decrease of total leukocyte, eosinophil, basophile, monocyte and lymphocyte. None of the techniques were effective to withdraw the Aldicarb compound from the organism.
\end{abstract}

Keywords: dog, carbamate, poisoning, dialysis techniques, haematology.

* Escola de Veterinária, Universidade Federal de Minas Gerais, Av. Antônio Carlos 6627, Cep 31270-010, Belo Horizonte, Minas Gerais, ptativet@yahoo.com

** Professora Adjunta do Departamento de Medicina Veterinária, Universidade Estadual de Maringá, Campus de Umuarama, PR.

*** Instituto de Ciências Biológicas, Universidade Federal de Minas Gerais, Av. Antônio Carlos, 6627, Pampulha, Belo Horizonte, Minas Gerais 


\section{Introdução}

Os carbamatos são ésteres do ácido carbâmico e sua ação tóxica sobre os animais é conseqüência dos efeitos sobre a inibição da acetilcolinesterase (ACHe) (Mahashur et al., 1994; Kwong, 2001). Cães intoxicados apresentam manifestações clínicas quando $50 \%$ ou mais da reserva de $\mathrm{ACHe}$ é inibida (Andrade Filho e Romano, 2001), com conseqüente hiperestimulação dos receptores muscarínicos, nicotínicos e do sistema nervoso central. O início do aparecimento dos sinais ocorre entre minutos e horas após a exposição, dependendo da via de contaminação, da quantidade de tóxico, da natureza do composto e da necessidade de biotransformação, para a substância se tornar ativa. As primeiras manifestações clínicas relativas à intoxicação geralmente são muscarínicas, seguidas das manifestações nicotínicas. Em alguns casos, as manifestações nicotínicas ocorrem concomitantemente com as muscarínicas. Os sinais nicotínicos, quando presentes, refletem a gravidade da intoxicação, informação importante para o clínico que deve, portanto, intervir imediatamente (Henry e Wiseman, 1998).

O diagnóstico de uma intoxicação aguda é baseado na história de exposição recente ao carbamato, sendo que o início dos sinais clínicos revela uma exposição ao carbamato há 12 horas, aproximadamente. Observa-se rápida melhora dos sinais clínicos com a administração de sulfato de atropina e de benzodiazepínicos (Mortensen, 1986; Bardin et al., 1994).

Como o Aldicarb é um dos pesticidas mais tóxicos que se conhece, atualmente vem sendo muito utilizado para a prática criminosa de extermínio de cães e gatos. Apesar da existência de medicamentos que controlam os efeitos muscarínicos e nicotínicos, e tratamento suporte para os demais sintomas, a substância, mesmo em pequenas doses, é altamente tóxica ao organismo, revelando alto índice de letalidade.

Tanto a hemodiálise como a hemoperfusão são procedimentos propostos para depuração sangüínea de várias substâncias tóxicas. A hemoperfusão é mais eficaz que a hemodiálise na depuração do sangue, com relação a fármacos ligados a proteínas, pois o carvão ativado, presente no hemoperfusor, compete com as proteínas plasmáticas, pela interação dos fármacos, adsorvendo e removendo-os da circulação (Schreiner, 1982).

Diante deste contexto, este experimento teve como objetivo avaliar o comportamento clínico e o perfil hematológico de cães intoxicados experimentalmente com carbamato (Aldicarb) e submetidos à hemodiálise e à hemoperfusão.

\section{Material e métodos}

Quinze cães adultos machos, saudáveis, sem raça definida, com peso médio de $23,29 \mathrm{~kg}$, oriundos do Centro de Zoonoses da Prefeitura de Belo Horizonte, receberam $30 \mathrm{mg} / \mathrm{kg}$ de peso vivo, de um produto vendido ilegalmente, conhecido popularmente como "chumbinho", adquirido em um estabelecimento comercial especializado em produtos agropecuários e analisado por cromatografia líquida (HPLC) segundo Lacassie et al. (2001), contendo 16,57\% de Aldicarb. Portanto, a dose correspondeu à intoxicação de $4,97 \mathrm{mg} / \mathrm{kg}$ de peso vivo de Aldicarb. O produto administrado por via oral, de forma espontânea, misturado à ração comercial do tipo úmida para cães ${ }^{1}$ foi totalmente consumido, garantindo a ingestão da dose proposta.

Este experimento foi realizado nas dependências do Hospital Veterinário da Escola de Veterinária da UFMG.

Todos os animais, para controle dos efeitos clínicos provocados pela droga, foram medicados 30 minutos e uma hora após contaminação, com sulfato de atropina ${ }^{2}$ e com benzodiazepínico, ${ }^{3}$ ambos na dose de $1,0 \mathrm{mg} / \mathrm{kg}$. Esses cães foram divididos em três grupos experimentais, compostos de cinco animais cada. Os animais do grupo I serviram de controle, não tendo sido submetidos a nenhum tratamento dialítico. Os animais do grupo II e do grupo III, três horas após administração do carbamato (Aldicarb), foram submetidos a sessões de duas horas de hemodiálise e de hemoperfusão, respectivamente.

Para realização das técnicas dialíticas (hemodiálise e hemoperfusão) foi feita implantação cirúrgica de cateter do tipo duplo-lúmen ${ }^{4}$ na veia jugular externa.

Durante a hemodiálise os animais permaneceram em decúbito lateral sobre uma mesa com colchão de espuma. $\mathrm{O}$ volume do fluxo sangüíneo foi de $80 \mathrm{~mL} /$ minuto e do dialisado de $500 \mathrm{~mL} /$ minuto. Foi necessária contenção química com benzodiazepínico, ${ }^{3}$ na dose de $1,0 \mathrm{mg} / \mathrm{kg}$, via endovenosa, a cada hora de diálise. A máquina de hemodiálise utilizada foi uma do tipo proporção, modelo $2008-\mathrm{C},{ }^{5}$ acoplada a uma unidade portátil de tratamento de água modelo WTU $100,{ }^{5}$ capaz de produzir água ultrapura pelo sistema de osmose reversa. Foi utilizado um hemodializador ${ }^{5}$ do tipo fibras ocas, alto fluxo, com membrana sintética de polissulfona, com área interna de $0,7 \mathrm{~m}^{2}$. As linhas de sangue ${ }^{5}$ utilizadas foram de tamanho pediátrico, linha arterial e venosa. A anticoagulação do sistema foi feita com heparina sódica ${ }^{6}$ na dose de $100 \mathrm{Ul} / \mathrm{kg}$, a cada 30 minutos de diálise.

Durante a hemoperfusão os animais permaneceram em decúbito lateral sobre uma mesa com colchão de espuma. 0 volume de fluxo sangüíneo foi de $50 \mathrm{~mL} /$ minuto. Foi necessária a contenção química utilizando benzodiazepínico ${ }^{3}$ (1,0mg/kg via endovenosa a cada uma hora do procedimento). A máquina de hemodiálise, utilizada para realização da hemoperfusão, foi a mesma mencionada anteriormente, do tipo proporção modelo 2008-C. ${ }^{5}$ O hemoperfusor ${ }^{7}$ utilizado era composto de $150 \mathrm{~g}$ de grânulos de carvão ativado revestido de celulose, com grande capacidade adsortiva, com $245 \mathrm{~mm}$ de comprimento, $87 \mathrm{~mm}$ de diâmetro, pesando $0,9 \mathrm{~kg}$, proporcionando $150000 \mathrm{~m}^{2}$ de área superficial e volume de preenchimento de $140 \mathrm{~mL}$ (Adsorba 150). As linhas de sangue ${ }^{5}$ utilizadas eram de tamanho pediátrico, tendo as linhas arteriais e venosas os volumes internos de $65 \mathrm{~mL}$ e $45 \mathrm{~mL}$ ("priming"), respectivamente. Para evitar a coagulação do sangue durante o procedimento, foi aplicada heparina sódica, ${ }^{5} \mathrm{com}$ dose inicial de $200 \mathrm{UI} / \mathrm{kg}$, e dose de $100 \mathrm{Ul} / \mathrm{kg}$, a cada 30 minutos de diálise, até o término do processo.

\footnotetext{
1 Ração úmida para cães adultos - Pedigree.

2 Sulfato de Atropina 1\% - Fraga.

${ }^{3}$ Diazepam - União Química.

4 Cateter Duplo-lúmen - Niagara.

${ }^{5}$ Fresenius Medical Care.

6 Heparina sódica - Heparin.

7 Adsorba 150 - Gambro Renal Products.

${ }^{8}$ Animal Blood Counter-Pack-ABX.
} 
Os animais foram submetidos a exames clínicos durante todo o experimento, sendo feitas as seguintes avaliações: estado geral, auscultação pulmonar, alterações musculares, presença de vômito, defecação e micção e alteração de comportamento.

Foram coletadas amostras de sangue com anticoagulante EDTA 10\% (sal dissódico do ácido etilenodiamino tetracético) para realização do hemograma conforme Jain (1993). As amostras foram coletadas nos seguintes tempos (T): T1 (tempo zero ou basal); T2 - 12 horas após colocação do cateter e antes da intoxicação; T3 - 30 minutos após intoxicação e antes do primeiro tratamento padrão (sulfato de atropina e benzodiazepínico); T4 - três horas após intoxicação e duas horas após a segunda aplicação do tratamento padrão (antes da hemodiálise no grupo II e da hemoperfusão no grupo III); T5 - cinco horas após a intoxicação e quatro horas após a segunda aplicação do tratamento padrão (depois da hemodiálise no grupo II e depois da hemoperfusão no grupo III).

O hemograma e contagem de plaquetas foram processados eletronicamente, ${ }^{8}$ e a contagem diferencial de leucócitos realizada em esfregaços sangüíneos corados pelo MayGrunwald-Giemsa de acordo com Jain (1993).

Também foi coletado sangue sem anticoagulante para obtenção do soro e urina antes da intoxicação; 30 minutos após a intoxicação e antes do tratamento padrão; três horas após a intoxicação e antes das técnicas dialíticas e cinco horas após a intoxicação, ou seja, imediatamente após as técnicas dialíticas. As amostras de dialisato foram colhidas antes e após a sessão de hemodiálise. Foi retirado o carvão ativado do hemoperfusor e analisado. Em todas essas amostras, pesquisou-se o Aldicarb pela técnica de Cromatografia Líquida de alta Eficiência (HPLC) segundo Lacassie et al. (2001).

O delineamento experimental adotado foi inteiramente casualizado com parcelas subdivididas no tempo. Os dados de hemograma foram comparados pelo teste $t$ de Student entre os grupos e tempos, segundo Sampaio (1998).

Este experimento foi aprovado pelo Comitê de Ética em Experimentação Animal (CETEA) da UFMG (protocolo 055/03). O destino final dos animais foi a adoção.

\section{Resultados e discussão}

Todos os animais do grupo I apresentaram sinais clínicos de sialorréia e fasciculações, iniciando cerca de 15 a 30 minutos após intoxicação. As manifestações nicotínicas ocorreram concomitantemente com as muscarínicas, assim como observadas por Andrade Filho et al. (2001). Delaunois et al. (1997) descrevem que os primeiros sintomas pós-intoxicação são muscarínicos, seguidos dos nicotínicos. Entretanto, Lifshitz (1994) afirmou que há a possibilidade da inversão da ordem do aparecimento dos sintomas. Cerca de 15 a 20 minutos após a intoxicação os animais começaram a defecar para, em seguida, apresentarem forte tenesmo e defecação intensa. Entre 45 a 60 minutos após a intoxicação, observouse a presença de secreção pulmonar, intensificando gradativamente e sendo corrigida após a segunda administração de sulfato de atropina e benzodiazepínico.

Para que o Aldicarb pudesse estar presente na corrente sangüínea dos animais intoxicados, durante os procedimen- tos dialíticos, grande quantidade deste composto foi administrada. Como a intoxicação provocada foi considerada grave, havendo risco iminente de óbito dos animais estudados, a primeira aplicação de sulfato de atropina e de benzodiazepínico foi realizada 30 minutos após intoxicação. O controle dos efeitos muscarínicos e nicotínicos, obtido pela aplicação de sulfato de atropina e de benzodiazepínico, não interfere na eficiência das técnicas dialíticas propostas, visto que o sulfato de atropina apenas provoca antagonismo de receptor com a acetilcolina e os benzodiazepínicos, alívio da inquietação, evitando também alguns sintomas do sistema nervoso central.

O sulfato de atropina deve ser aplicado até que os sinais dos efeitos muscarínicos desapareçam, podendo ser repetida de 15 a 30 minutos como indicado por Delaunois et al., 1997. Lifshitz (1994), tratando crianças intoxicadas por carbamato, admitidas no CTI do Centro Médico de Sorocaba, aplicou sulfato de atropina em intervalos de 5 a 10 minutos, até desaparecerem os sintomas muscarínicos.

Cerca de 10 minutos após administração da primeira aplicação de sulfato de atropina e de benzodiazepínico, observou-se a diminuição da sialorréia e da defecação. A secreção pulmonar manteve-se acentuada em três animais, diminuindo após administração do tratamento. Um dos animais não apresentou secreção pulmonar. Dois deles ficaram muito agitados, vocalizando, com discretos movimentos de pedalagem.

Após uma hora da intoxicação foi realizada a segunda aplicação de sulfato de atropina e de benzodiazepínico, quando se observou que dois animais ficaram prostrados durante aproximadamente uma hora. Outros três animais ficaram mais agitados, agravando os sintomas de vocalização. Esses mesmos três animais mantiveram, ainda, sinais de fasciculação, incoordenação motora, secreção nasal e dificuldade respiratória com dispnéia.

Três horas após a intoxicação foi novamente administrado benzodiazepínico. Após esse procedimento, dois animais ficaram agitados e os outros três ficaram prostrados. Neste momento do experimento, o quadro clínico de um animal revelou um grave edema pulmonar, evoluindo para parada respiratória com paralisia dos músculos torácicos, coma e óbito. A morte ocorreu por insuficiência respiratória, constrição brônquica, secreção pulmonar e paralisia dos músculos torácicos conforme descrito por Baron (1991).

Cinco horas após a intoxicação, os animais não apresentaram desejo de beber água ou alimentar-se. Doze horas depois estavam mais dispostos, porém revelando incoordenação motora, com dificuldade de locomoção e certa rigidez muscular dos membros.

Os animais do grupo II que foram submetidos à hemodiálise apresentaram início dos sinais clínicos cerca de 10 a 15 minutos após intoxicação. Dois animais apresentaram inicialmente defecação e micção, sendo que os outros três iniciaram com intensa sialorréia. Dificuldade respiratória e tosse foram observadas em dois animais, sendo que estes permaneceram em decúbito esternal para facilitar a respiração. Dois cães apresentaram secreção pulmonar 40 minutos após intoxicação. Dos cinco animais, dois vomitaram antes da primeira aplicação de sulfato de atropina e do benzodiazepínico; dois imediatamente após e um animal não vomitou. Três animais apresentaram fasciculações 20 
minutos após intoxicação; um, 60 minutos após intoxicação e o outro durante a sessão de hemodiálise.

Pôde-se observar que os sinais de sialorréia apareceram em torno de 15 minutos após a intoxicação, cessando após a primeira aplicação de sulfato de atropina e do benzodiazepínico. Vômito, defecação e micção foram intensos, e um animal apresentou diarréia com presença de sangue. Secreção brônquica, lacrimejamento e sialorréia também foram observados. Esses sinais são também relatados por Melo e Silva Junior (2006) em cães, que relataram que os principais efeitos muscarínicos são sialorréia, vômitos e diarréia.

Após a primeira administração de sulfato de atropina e de benzodiazepínico, sialorréia, defecação e vômito foram diminuindo até cessarem. As fasciculações continuaram aumentando de intensidade. Após a segunda aplicação de sulfato de atropina e de benzodiazepínico, os animais permaneceram mais tranqüilos e as fasciculações ficaram mais brandas. Apenas um animal apresentou discretos movimentos de pedalagem.

Todos os animais foram colocados em decúbito lateral para o início da sessão de hemodiálise. Durante a sessão, dois animais apresentaram vômito. Após o término, dois animais permaneceram prostrados, recuperando cerca de oito horas depois. Os outros três ficaram mais dispostos, beberam água logo após o procedimento, sendo que um deles alimentou-se.

Nos animais do grupo da hemoperfusão (GIII) os primeiros sintomas apareceram entre 10 e 30 minutos após a intoxicação. Dois apresentaram defecação intensa após a intoxicação, apresentando diarréia com presença de sangue. Os demais apresentaram defecação mais tardiamente. Em um dos animais deste grupo, fasciculações apareceram em torno de 15 minutos após intoxicação e, após 30 minutos, em três deles. Foi observada dificuldade respiratória em três animais, aproximadamente 30 minutos após a intoxicação. Sialorréia intensa e vômitos foram observados em todos os cães deste grupo cerca de 15 minutos após a intoxicação.

Os sinais de alteração respiratória foram se agravando próximos ao momento da primeira aplicação de sulfato de atropina e de benzodiazepínico. Os animais tinham dificuldade de se manter em posição ortostática, com incoordenação e tremores musculares.

Cerca de cinco minutos após a primeira aplicação de sulfato de atropina e do benzodiazepínico, cessaram-se os sinais de vômito e diarréia. Dois animais apresentaram secreção pulmonar com sinais de dificuldade respiratória, progredindo à cianose.

Após a segunda aplicação do tratamento padrão, dois animais apresentaram episódios de pedalagem e tentativa de levantar, sendo que os outros ficaram prostrados. As fasciculações ficaram mais intensas em dois animais.

Após o início da sessão de hemoperfusão dois animais ficaram muito agitados possivelmente devido à retirada do benzodiazepínico pela técnica de hemoperfusão. Os outros permaneceram em decúbito lateral, sob efeito da sedação. Terminada a hemoperfusão, três animais mostraram-se mais dispostos, sendo que um deles chegou a alimentar. Os outros recuperaram cerca de 12 horas após a sessão.

Como não foram observadas diferenças no perfil clínico entre os animais dos três grupos, provavelmente os tratamentos dialíticos (hemodiálise e hemoperfusão) não extraíram o Aldicarb.

A Tabela 1 mostra a concentração de Aldicarb (ppm) no soro dos cães intoxicados e submetidos a hemodiálise e hemoperfusão em diferentes tempos.
Tabela 1: Pesquisa de Aldicarb (ppm) no soro de cães intoxicados e tratados com atropina e benzodiazepínico - tratamento padrão (Grupo I), tratamento padrão e hemodiálise (Grupo II), tratamento padrão e hemoperfusão (Grupo III) em diferentes tempos

\begin{tabular}{c|c|c|c}
\hline Grupo I /Animal & $\begin{array}{c}\text { 30min após a } \\
\text { intoxicação }\end{array}$ & $\begin{array}{c}\text { 3h após a } \\
\text { intoxicação e antes } \\
\text { das técnicas dialíticas }\end{array}$ & $\begin{array}{c}\text { 5h após a } \\
\text { intoxicação e após } \\
\text { as técnicas dialíticas }\end{array}$ \\
\hline 1 & 0,0031 & não detectado & 0,0064 \\
\hline 2 & 0,0139 & 0,0580 & *óbito \\
\hline 3 & não detectado & traços & não detectado \\
\hline 4 & traços & traços & não detectado \\
\hline 5 & não detectado & não detectado & não detectado \\
\hline Grupo II /Animal & & & não detectado \\
\hline 1 & traços & não detectado & não detectado \\
\hline 2 & não detectado & não detectado & 0,0060 ppm \\
\hline 3 & 0,0050 ppm & não detectado & não detectado \\
\hline 4 & não detectado & não detectado & não detectado \\
\hline 5 & não detectado & não detectado & não detectado \\
\hline Grupo III/Animal & & & não detectado \\
\hline 1 & 0,0100 ppm & não detectado & não detectado \\
\hline 2 & 0,0100 ppm & não detectado & não detectado \\
\hline 3 & não detectado & não detectado & não detectado \\
\hline 4 & não detectado & não detectado & \\
\hline 5 & não detectado & não detectado & \\
\hline
\end{tabular}

No soro do animal 1 do grupo I, 30 minutos após a intoxicação, foi identificado 0,0031ppm de Aldicarb, mostrando a rápida absorção e distribuição desta substância (Tabela 1). Neste momento o animal ainda não havia vomitado, vindo a apresentar vômito cinco minutos após a colheita de material. Porém, na segunda colheita, três horas após a intoxicação, não foi detectada a presença de Aldicarb no soro. Entretanto, na última colheita, foi detectado 0,0064ppm. Isso pode ser explicado pela baixa concentração da substância na circulação, muito próximo do limite mínimo de detecção da técnica utilizada. O limite de detecção é o valor mínimo em que a substância aparece nos resultados e varia de acordo com a substância. Neste trabalho, o limite de detecção foi de 0,0031ppm, portanto valores abaixo foram considerados traços.

No animal 2 do grupo I (Tabela1), observou-se no soro a presença de 0,0139ppm de Aldicarb e na segunda colheita, três horas após a intoxicação, esse nível aumentou para 0,0580ppm. Este animal não apresentou episódios de vômitos, vindo a óbito três horas após a intoxicação. Baron (1991) relatou que as doses letais de Aldicarb são: para ratos $(0,46$ a 1,23 $\mathrm{mg} / \mathrm{kg})$, camundongos $(0,38$ a $1,50 \mathrm{mg} / \mathrm{kg})$, suínos $(1,0 \mathrm{mg} /$ $\mathrm{kg})$ e coelhos $(1,3 \mathrm{mg} / \mathrm{kg})$.

Nos animais 3 e 4 do grupo I somente foram detectados traços de Aldicarb três horas após a intoxicação e no animal 5 não foi detectado Aldicarb nos tempos estudados. 
No grupo II, 30 minutos após a intoxicação, os animais 1 e 3 apresentaram traços e 0,0050ppm de Aldicarb, respectivamente e no animal 3, foi detectado 0,0060ppm após a hemodiálise. Já nos animais do grupo III, somente foi detectado Aldicarb (0,00100ppm) nos animais 1 e 2, 30 minutos após intoxicação, não sendo detectado nos demais em tempo algum (Tabela1).

As variações observadas de detecção e não detecção de Aldicarb no soro podem ser justificadas por uma baixa concentração da substância na circulação, por fatores individuais relacionados à absorção, distribuição, metabolismo e eliminação de fármacos, bem como pelos episódios de vômitos apresentados por alguns animais, quando houve eliminação de parte do Aldicarb administrado, como mencionado por Chuecos et al. (1992).

Tanto a hemodiálise quanto a hemoperfusão são técnicas de depuração sangüínea, porém não se mostraram eficientes para a remoção do aldicarb. Além disso, a técnica não é capaz de remover partículas que estejam ligadas às proteínas plasmáticas, nem apresenta boa capacidade dialítica em situações em que uma substância esteja com baixas concentrações plasmáticas. A hemodiálise depende de um gradiente de concentração maior para o meio sangue, e sua eficácia aumenta quando este gradiente de concentração é máximo (Daugirdas e Van Stone, 2003; Winchester e Kitiyakara, 2003; Veado, 2003). A hemoperfusão, por outro lado, tem a capacidade de remover grandes partículas, mesmo aquelas ligadas a proteínas, porém, como a hemodiálise, apresenta a limitação da concentração da substância presente na corrente sangüínea. Schreiner (1982) afirmou que para se utilizar a técnica de hemoperfusão, a molécula-alvo deve estar presente no sangue em concentração suficiente para produzir diferença no equilíbrio entre célula e tecido. Se a moléculaalvo está ligada, o solvente deve ter melhor ligação energética, ou melhor, afinidade que as células ou proteínas sangüíneas. Assim, a influência da distribuição da substância no organismo é determinante para o sucesso das técnicas. Aquele agente que, ao penetrar no organismo apresenta uma distribuição rápida, atingindo seus sítios de ação e apresentando-se com baixas concentrações na corrente sangüínea, não será bem dialisado (Daugirdas e Van Stone, 2003; Winchester e Kitiyakara, 2003; Veado, 2003).

Embora os animais tenham recebido uma dose suficiente de Aldicarb para causar intoxicação, esse não foi detectado na corrente sangüínea nem antes da hemodiálise como da hemoperfusão, o que pode ter comprometido a avaliação da capacidade das técnicas de depuração da substância. Desta forma, os resultados obtidos das análises do dialisato e do carvão ativado do hemoperfusor, mostram coerência, por não apresentarem Aldicarb em suas amostras, após hemodiálise e hemoperfusão.

Nos casos de acidentes em que os animais ingerem grandes quantidades de carbamatos, o vômito promove, muitas vezes, a eliminação de grande parte desta substância, reduzindo a quantidade final absorvida. Aqueles animais que não a eliminam pelo vômito, têm grande possibilidade de vir à óbito, devido aos efeitos graves por ela provocado.

Estima-se que a quantidade de "chumbinho" fornecida aos animais foi próxima à oferecida em situações de envenenamento intencional, ou ingerida em envenenamentos aciden- tais. Sendo assim, a indicação da realização da hemodiálise ou hemoperfusão torna-se questionável. A eliminação do composto Aldicarb é rápida, cerca de 70 a $80 \%$ de uma dose única é excretada na urina nas primeiras 24 horas (Delaunois et al., 1997). Entretanto não foi detectado Aldicarb nas amostras de urina dos animais. Possivelmente, até o último momento da coleta da urina (5h), o Aldicarb não tenha sido eliminado. Estes resultados corroboram os resultados clínicos relatados anteriormente, da não utilização de técnicas de hemodiálise e hemoperfusão, para cães intoxicados com dose estimada de Aldicarb de $4,97 \mathrm{mg} / \mathrm{kg}$ de peso vivo para retirada do mesmo do organismo animal.

Com relação ao quadro hematológico, possivelmente, as diferenças significativas encontradas no eritrograma, pela análise dos valores do número de hemácias, concentração de hemoglobina e hematócrito entre o T1 e T2 nos grupos I e Il foi devido a implantação cirúrgica do cateter, que pode causar discretas hemorragias (Tabela 2).

Após intoxicação (30 minutos), houve aumento relativo dos valores do eritrograma (Pd"0,05) nos grupos II e III em T3, quando comparados ao T2, revelando hemoconcentração, possivelmente, pela perda de líquido nos vômitos, diarréia e miccção acentuada presentes em todos os animais destes grupos, ou ainda pelo efeito da contração esplênica (estresse adrenérgico). Esses valores apresentaram tendência para retornar aos valores anteriores, porém no grupo II (hemodiálise) em T5 percebe-se novamente uma hemoconcentração, justificada pela perda de líquidos durante o processo hemodialítico. Todos os valores médios de plaquetas se encontraram dentro dos valores de referência para a espécie canina. Após implantação do cateter ocorreu uma diminuição estatisticamente significativa $(P d " 0,05)$ no valor médio das plaquetas nos grupos I e II (Tabela 2).

Em relação aos índices hematimétricos, o volume corpuscular médio (VCM) não apresentou nenhuma alteração entre grupos nos diferentes tempos. Hemoglobina corpuscular média (HCM) e concentração de hemoglobina corpuscular média (CHCM) apresentaram aumento dos seus valores médios apenas no T2 do grupo II (Tabela 2), sem significado clínico.

Trinta minutos após intoxicação houve aumento do número total de leucócitos nos três grupos estudados, sendo significativo (Pd"0,05) nos grupos II e III. O aumento significativo no número absoluto de neutrófilos foi observado nos grupos II e III (Tabela 3). Possivelmente esta leucocitose foi fisiológica por liberação de células do pool marginal e induzida pelo estresse e pela dor (Lopes et al., 1996). Caldas (2000) também observou leucocitose nos pacientes após intoxicação aguda por carbamato.

Após a colocação do cateter houve um aumento significativo do número absoluto de neutrófilos bastonetes no grupo I. Porém, 30 minutos após a intoxicação o aumento dos bastonetes somente foi significativo no grupo III. Ressalta-se que todos os animais, mesmo no tempo basal apresentaram valores de neutrófilos bastonetes acima dos limites normais para a espécie de acordo com Ferreira Neto et al. (1981). Quando se comparam os grupos, não houve diferença nos vaalores médios após a intoxicação e nem mesmo após os tratamentos realizados (Tabela 3). 
Tabela 2: Valores médios de hemácias $\left(x 10^{6} / \mu \mathrm{L}\right)$, hemoglobina (g/dL), hematócrito (\%), VCM (fL), HCM (pg), $\mathrm{CHCM}(\%)$ e plaquetas (x103/iL) de cães intoxicados com Aldicarb e tratados com sulfato de atropina e benzodiazepínico - tratamento padrão (grupo I), tratamento padrão e hemodiálise (grupo II) e tratamento padrão e hemoperfusão (grupo III)

\begin{tabular}{|c|c|c|c|c|c|c|}
\hline & \multirow[b]{3}{*}{ Grupos } & \multicolumn{5}{|c|}{ Tempos } \\
\hline & & T1 & T2 & T3 & T4 & T5 \\
\hline & & Zero & $\begin{array}{l}\text { 12h após implantação do } \\
\text { cateter e antes da } \\
\text { intoxicação }\end{array}$ & $\begin{array}{l}30 \text { min após a } \\
\text { intoxicação }\end{array}$ & $\begin{array}{c}\text { 3 h após a intoxicação } \\
\text { (antes das técnicas } \\
\text { dialíticas) }\end{array}$ & $\begin{array}{c}\text { 5h após a intoxicação } \\
\text { (depois das técnicas } \\
\text { dialíticas) }\end{array}$ \\
\hline & 1 & $6,94_{\mathrm{Aa}}$ & $6,09_{\mathrm{Ba}}$ & $6,52_{\text {Aba }}$ & $6,83_{\text {Aba }}$ & $6,26_{\mathrm{Aba}}$ \\
\hline Hemácias & II & $6,32_{\mathrm{Aba}}$ & $5,08_{\mathrm{Ca}}$ & $6,40_{\text {Aba }}$ & $5,66_{\mathrm{Bca}}$ & $6,49_{\text {Aa }}$ \\
\hline \multirow[t]{2}{*}{$\left(\times 10^{6} / \mu \mathrm{l}\right)$} & III & $5,97_{\mathrm{ABa}}$ & $5,48_{\mathrm{Ba}}$ & $6,55_{\mathrm{Aa}}$ & $5,47_{\mathrm{Ba}}$ & $5,54_{\mathrm{Ba}}$ \\
\hline & $\mathrm{I}$ & $15,50_{\mathrm{Aa}}$ & $13,48_{\mathrm{Ba}}$ & $14,48_{\mathrm{ABa}}$ & $15,02_{\text {Aba }}$ & $14,06_{\mathrm{ABa}}$ \\
\hline Hemoblobina & II & $13,78_{\text {АВа }}$ & $11,78_{\mathrm{Ca}}$ & $13,98_{\mathrm{Aa}}$ & $12,16_{\text {вса }}$ & $13,88_{\mathrm{ABa}}$ \\
\hline \multirow[t]{2}{*}{$(g / d l)$} & III & $13,94_{\text {АBa }}$ & $12,72_{\mathrm{Ba}}$ & $15,26_{\mathrm{Aa}}$ & $12,62 \mathrm{Ba}$ & $12,88_{\mathrm{Ba}}$ \\
\hline & $\mathrm{I}$ & $46,54_{\mathrm{Aa}}$ & $40,92_{\text {Ва }}$ & $43,90_{\mathrm{ABa}}$ & $45,96_{\text {Aba }}$ & $42,18_{\text {Aba }}$ \\
\hline Hematócrito & II & $41,72 A_{\mathrm{Ba}}$ & $34,30_{\mathrm{Ca}}$ & $42,62_{\mathrm{Aa}}$ & $37,20_{\text {Bса }}$ & $42,70_{\mathrm{Aa}}$ \\
\hline \multirow[t]{2}{*}{$(\%)$} & III & $41,16_{\text {АВa }}$ & $37,78_{\mathrm{Ba}}$ & $45,48_{\mathrm{Aa}}$ & $37,50_{\mathrm{Ba}}$ & $37,94_{\mathrm{Ba}}$ \\
\hline & 1 & $67,2_{\mathrm{Aa}}$ & $67,0_{\mathrm{Aa}}$ & $67,2_{\mathrm{Aa}}$ & $67,4_{\mathrm{Aa}}$ & $67,2_{\mathrm{Aa}}$ \\
\hline VCM & ॥ & $66,0_{\mathrm{Aa}}$ & $68,2_{\mathrm{Aa}}$ & $66,6_{\mathrm{Aa}}$ & $66,0_{\mathrm{Aa}}$ & $66,0_{\mathrm{Aa}}$ \\
\hline \multirow[t]{2}{*}{$(\%)$} & III & $69,2_{\mathrm{Aa}}$ & $69,2_{\mathrm{Aa}}$ & $69,6_{\mathrm{Aa}}$ & $68,8_{\mathrm{Aa}}$ & $68,8_{\mathrm{Aa}}$ \\
\hline & $\mathrm{I}$ & $22,40_{\mathrm{Aa}}$ & $22,24_{\mathrm{Aa}}$ & $22,24_{\mathrm{Aa}}$ & $21,96_{\mathrm{Aa}}$ & $22,46_{\mathrm{Aa}}$ \\
\hline $\mathrm{HCM}$ & ॥ & $21,74_{\mathrm{Ba}}$ & $23,80_{\mathrm{Aa}}$ & $21,78_{\text {ва }}$ & $21,52_{\mathrm{Ba}}$ & $21,48_{\mathrm{Ba}}$ \\
\hline \multirow[t]{2}{*}{ (ppg) } & III & $23,54_{\mathrm{Aa}}$ & $23,40_{\mathrm{Aa}}$ & $23,26_{\mathrm{Aa}}$ & $23,38_{\mathrm{Aa}}$ & $23,56_{\mathrm{Aa}}$ \\
\hline & $\mathrm{I}$ & $33,32_{\mathrm{Aa}}$ & $33,04_{\mathrm{Aa}}$ & $33,0_{\mathrm{Aa}}$ & $32,64_{\mathrm{Aa}}$ & $33,34_{\mathrm{Aa}}$ \\
\hline $\mathrm{CHCM}$ & II & $32,86_{\mathrm{Ba}}$ & $34,64_{\mathrm{Aa}}$ & $32,58_{\mathrm{Ba}}$ & $32,50_{\mathrm{Ba}}$ & $32,44_{\mathrm{Ba}}$ \\
\hline \multirow[t]{2}{*}{$(\%)$} & III & $33,94_{\mathrm{Aa}}$ & $33,76_{\mathrm{Aa}}$ & $33,46_{\mathrm{Aa}}$ & $33,84_{\mathrm{Aa}}$ & $34,18_{\mathrm{Aa}}$ \\
\hline & $\mathrm{I}$ & $364,0_{\mathrm{Aa}}$ & $238,2 \mathrm{Ba}$ & $262,6_{\mathrm{Ba}}$ & $280,6_{\mathrm{Ba}}$ & $266,6_{\mathrm{Ba}}$ \\
\hline \multirow{2}{*}{$\begin{array}{c}\text { Plaquetas } \\
\left(\times 10^{3} / \mu l\right)\end{array}$} & II & $285,6_{\text {Aab }}$ & $178,0_{\mathrm{Ca}}$ & $241,2_{\mathrm{ABa}}$ & $209,4_{\mathrm{BCa}}$ & $196,0_{\mathrm{BCa}}$ \\
\hline & III & $233,2_{\mathrm{Ab}}$ & $179,6_{\mathrm{Aa}}$ & $234,6_{\mathrm{Aa}}$ & $215,8_{\mathrm{Aa}}$ & $189,2_{\mathrm{Aa}}$ \\
\hline
\end{tabular}

Médias seguidas de mesmas letras maiúsculas não diferem estatisticamente entre os tempos para cada grupo (Pd"0,05) Médias seguidas de mesmas letras minúsculas não diferem estatisticamente entre os grupos para cada tempo(Pd"0,05)

Após a execução das técnicas dialíticas, não houve diferença significativa nos valores de leucócitos totais, apesar de se observar uma redução significativa (de 17.720/ $\mu \mathrm{L}$ para 13.200/ $\mu \mathrm{L}$ ) após a hemoperfusão (Tabela3). Massola (1995) também relatou diminuição dos leucócitos totais após a técnica da hemoperfusão. Todavia, Winchester e Kitiyakara (2003) relataram que os valores médios de leucócitos quando elevados na hemoperfusão retornam aos parâmetros de referência dentro de 24 a 48 horas.

De forma semelhante aos leucócitos totais, os valores absolutos de eosinófilos, basófilos, monócitos e linfócitos não apresentaram redução após a hemodiálise ou hemoperfusão.

\section{Conclusão}

A ingestão de $4,97 \mathrm{mg} / \mathrm{kg}$ de Aldicarb foi capaz de causar alterações clínicas em cães manifestadas por uma síndrome muscarínica, nicotínica e neurológica, alterações hematológicas, como hemoconcentração e leucocitose que não foram influenciadas pelas técnicas dialíticas.

\section{Referências}

ANDRADE FILHO, A.; ROMANO, C. Anticolinesterásicos. In: ANDRADE FILHO, A.; ROMANO, C.; DIAS, M.B. Toxicologia clínica. Belo Horizonte: Folium, 2001. p. 53-60.

BARDIN, P. G.; EEDEN, S. F.; MOOLMAN, J.A. et al. Organophosphate and carbamate poisonig. Arch. Intern. Med., v. 154, p. 1433-1441, 1994. BARON, R. L. Carbamate insecticides. In: HAYES JR., W. J.; LAWS JR., E. R. Handbook of pesticide toxicology. San Diego: Academic Press, 1991, v. 3, p. 1125-1189.

CALDAS, L. Q. A. Intoxicações exógenas agudas por carbamatos, organofosforados, compostos bipiridílicos e piretróides. Centro de Controle de Intoxicações de Niterói. Hospital Universitário Antônio Pedro - Universidade Federal Fluminesnse. Niterói, p. 1-39, 2000.

CHUECOS, J. M.; JURADO, M. D. C.; GIMENEZ, M. P. et al. Experience with hemoeprfusion for organophosphate poisoning. Crit. Care Med., v. 20, n. 11, p. 1538-1543, 1992.

DELAUNOIS, A.; LESSIRE, F.; FANAL, H. et al. Intoxications au Temik® chez les animaux domestiques et sauvages: un problème alarmant en Wallonie. Ann. Méd. Vét., v 141, p. 353-360, 1997. 


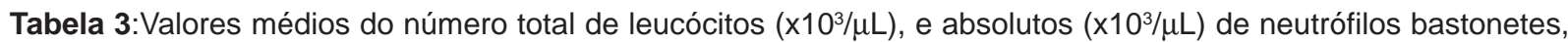
de neutrófilos segmentados, de eosinófilos, de basófilos, de monócitos e de linfócitos em cães intoxicados com Aldicarb e tratados com sulfato de atropina, benzodiazepínico (grupo I), hemodiálise (grupo II) e hemoperfusão (grupo III) em diferentes tempos

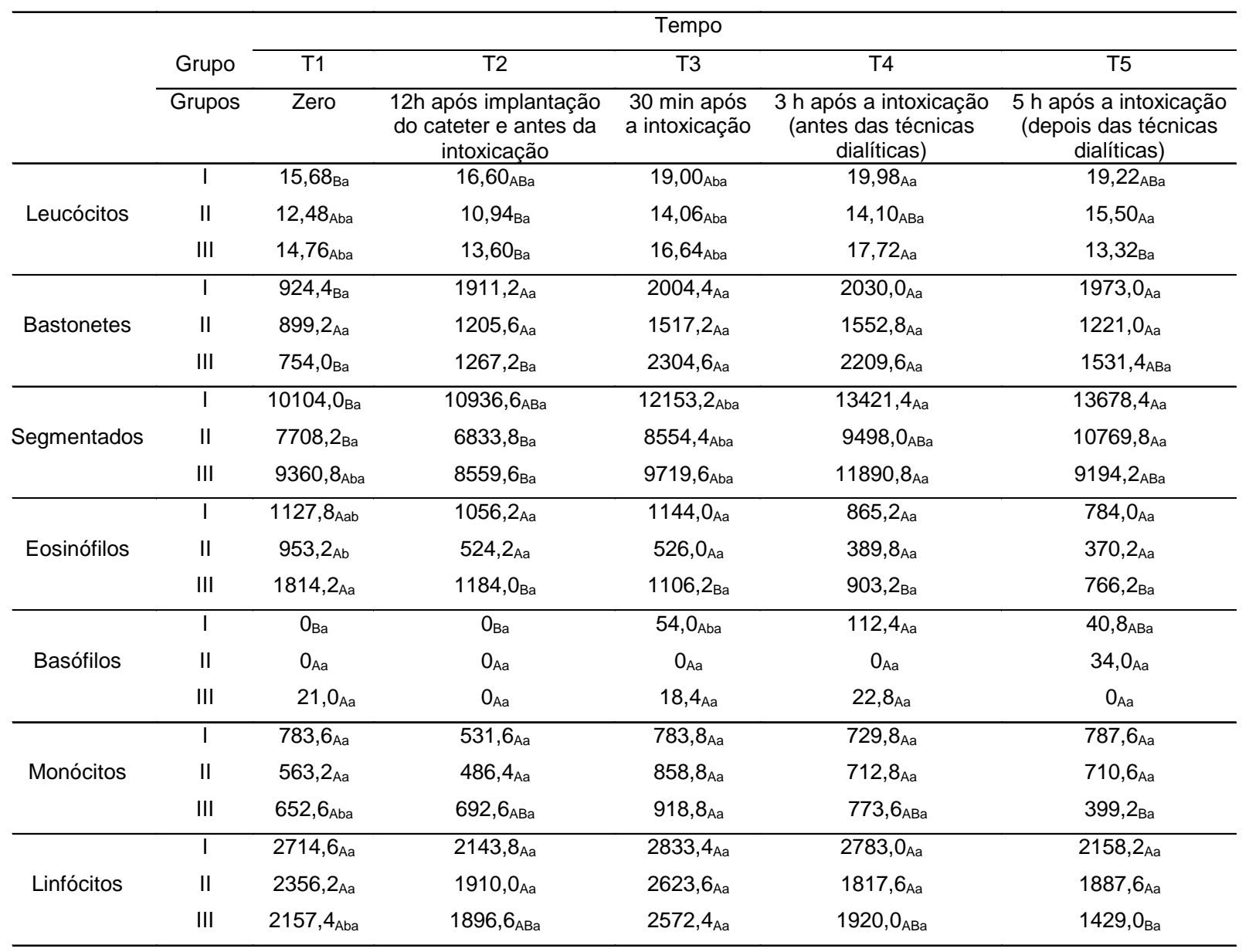

FERREIRA NETO, J. M.; VIANA, E. S; MAGALHÃES, L.M. Patologia clínica veterinária. Belo Horizonte: Rabelo, 1981. 279 p.

HENRY, J. A.; WISEMAN, H.M. Plaguicidas. In:_. . Tratamiento de las intoxicaciones. Ginebra: Organización Mundial de La Salud, 1998. p. 119-159.

JAIN, N. C. Essentials of veterinary hematology. Philadelphia: Lea \& Febiger, 1993, $417 \mathrm{p}$.

KWONG, T. C. Organophosphate and carbamate pesticide poisoning. In: SHAW, L. M.; KWONG, T. C.; ROSANO, T. G. et al. The clinical toxicology laboratory. Washington: AACC, 2001. p. 359-368.

LACASSIE, E., MARQUET, P. GAULIER, J. et al. Sensitive and specific multiresidue methods for determination of pesticides of various classes and forensic toxicology. For. Sci. Int., v. 121, p. 116-125, 2001.

LIFSHITZ, M.; ROTENBER, M.; SOFER, S. et al. Carbamate poisoning and oxime treatment in children: a clinical and laboratory study. Pediatrics, v. 93, n. 4, p. 652-655, 1994.

LOPES, S. T. A.; BIONDO, A. W.; CUNHA, C.M. S. et al. Patologia clínica veterinária. Santa Maria, 1996. $167 \mathrm{p}$.

MAHASHUR, A. A.; CHAUDHURI, A.; GOSWAMY, R. Study of respiratory failure in organophosphate and carbamate poisoning. Heart Lung, v. 23, n. 6, p. 466-472, 1994.
MASSOLA, V. C. Métodos dialíticos. In: CRUZ, J.; PRAXEDES, J.N.; CRUZ, H. M. M. Nefrologia. São Paulo: Sarvier, 1995, p. 201-226.

MELO, M. M.; SILVA JUNIOR, P.G. Intoxicações e envenenamentos. In: RABELO, R.C., CROWE JUNIOR D.T. Fundamentos de terapia intensiva veterinária em pequenos animais: condutas no paciente crítico. Rio de Janeiro: LS Livros Veterinária, 2005, cap. 62, p. 695-706.

MORTENSEN, M. L.; Management of acute childhood poisoning caused by selected insecticides and herbicides. Pediatr. Toxicol., v. 33, n. 2, p. 421-445, 1986.

SAMPAIO, I.B.M. Estatística aplicada à experimentação animal. Belo Horizonte: Fundação de Ensino e Pesquisa em Medicina Veterinária e Zootecnia-FEP-MVZ, 1998, 221 p.

SCHREINER, G. E. Clinical hemoperfusion. In:BONOMINI, V.; CHANG, T. M. S. (Eds.). Contributions to nephrology: hemoperfusion. Sidney: Satzstudio Frohberg, 1982. v. 29, p. 7-10.

VEADO, J. C. C.; Hemodiálise: por que empregar a técnica em animais. Medvep, v. 1,n. 1, p. 53-57, 2003.

WINCHESTER, J. F.; KITIYAKARA, C. Uso de diálise e de hemoperfusão no tratamento de intoxicações. In: DAUGIRDAS, J. T.; BLAKE, P. G.; ING, T. S. Manual de diálise. 3. ed. Rio de Janeiro: Medsi, 2003. p. 268283. 